\title{
Synthesis and reactivity of heptamethyl-cyclohexadienyl rhodium(III) complexes
}

Roman A. Pototskiya, Yulia V. Nelyubina ${ }^{a}$, Dmitry S. Perekalina, ${ }^{a, k}$

a Nesmeyanov Institute of Organoelement Compounds, Russian Academy of Sciences, 28 Vavilova, Moscow, 119991, Russian Federation. Tel.: +7 (499) 135 9367;

Fax: +7 (499) 135 5085. E-mail: dsp@ineos.ac.ru

b Plekhanov Russian University of Economics, 36 Stremyannyi pereulok, Moscow, 117997, Russian Federation.

\section{Supporting Information}

\section{Tables of context}

${ }^{1} \mathrm{H},{ }^{13} \mathrm{C},{ }^{31} \mathrm{P}$ NMR spectra:

4-methylene-1,1,2,3,5,6-hexamethylcyclohexadiene-2,5 (2)

$\left[\left(\eta^{5}-\mathrm{C}_{6} \mathrm{Me}_{7}\right) \mathrm{RhCl}_{2}\right]_{2}(4)$

$\left[\left(\eta^{6}-\mathrm{C}_{6} \mathrm{Me}_{6}\left(\mathrm{CH}_{2}\right)\right) \mathrm{Rh}\left(\mathrm{C}_{8} \mathrm{H}_{12}\right)\right]\left(\mathrm{PF}_{6}\right)(5)$ S4

$\left(n^{5}-\mathrm{C}_{6} \mathrm{Me}_{7}\right) \mathrm{Rh}(\mathrm{Py}) \mathrm{Cl}_{2}(6 \mathrm{a})$ S5

$\left(\eta^{5}-\mathrm{C}_{6} \mathrm{Me}_{7}\right) \operatorname{Rh}\left(\mathrm{P}(\mathrm{OEt})_{3}\right) \mathrm{Cl}_{2}(6 \mathrm{~b})$ S6-S7

$\left(\eta^{5}-\mathrm{C}_{6} \mathrm{Me}_{7}\right) \mathrm{Rh}\left(\mathrm{PPh}_{3}\right) \mathrm{Cl}_{2}(6 \mathrm{c})$ S8-S9

$\left[\left(\eta^{5}-C_{6} \mathrm{Me}_{7}\right) \mathrm{Rh}(\mathrm{dppe}) \mathrm{Cl}\right]\left(\mathrm{PF}_{6}\right)(7)$ S10-S11

$\left[\left(\eta^{5}-\mathrm{C}_{6} \mathrm{Me}_{7}\right) \operatorname{Rh}\left(\mathrm{C}_{6} \mathrm{Me}_{6}\right)\right]\left(\mathrm{BF}_{4}\right)_{2}(8)$

$\left(\eta^{5}-\mathrm{C}_{6} \mathrm{Me}_{7}\right) \mathrm{Rh}\left(\mathrm{C}_{6} \mathrm{H}_{4}-\mathrm{Py}\right) \mathrm{Cl}(9)$

Stoichiometric reaction of 9 with 3-hexyne

The supplemental file "Optimized structures.xyz" contains the computed Cartesian coordinates of all of the molecules reported in this study. The file may be opened as a text file to read the coordinates, or opened directly by a molecular modeling program such as Mercury (version 3.3 or later, http://www.ccdc.cam.ac.uk/pages/Home.aspx) for visualization and analysis. 
Figure S1. ${ }^{1} \mathrm{H}$ NMR spectrum of 4-methylene-1,1,2,3,5,6-hexamethylcyclohexadiene-2,5 $\mathrm{C}_{6} \mathrm{Me}_{6}\left(\mathrm{CH}_{2}\right)(\mathbf{2})$ in $\mathrm{CDCl}_{3}$

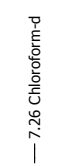<smiles>C=C1C(C)=C(C)C(C)(C)C(C)=C1C</smiles>

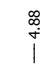

2

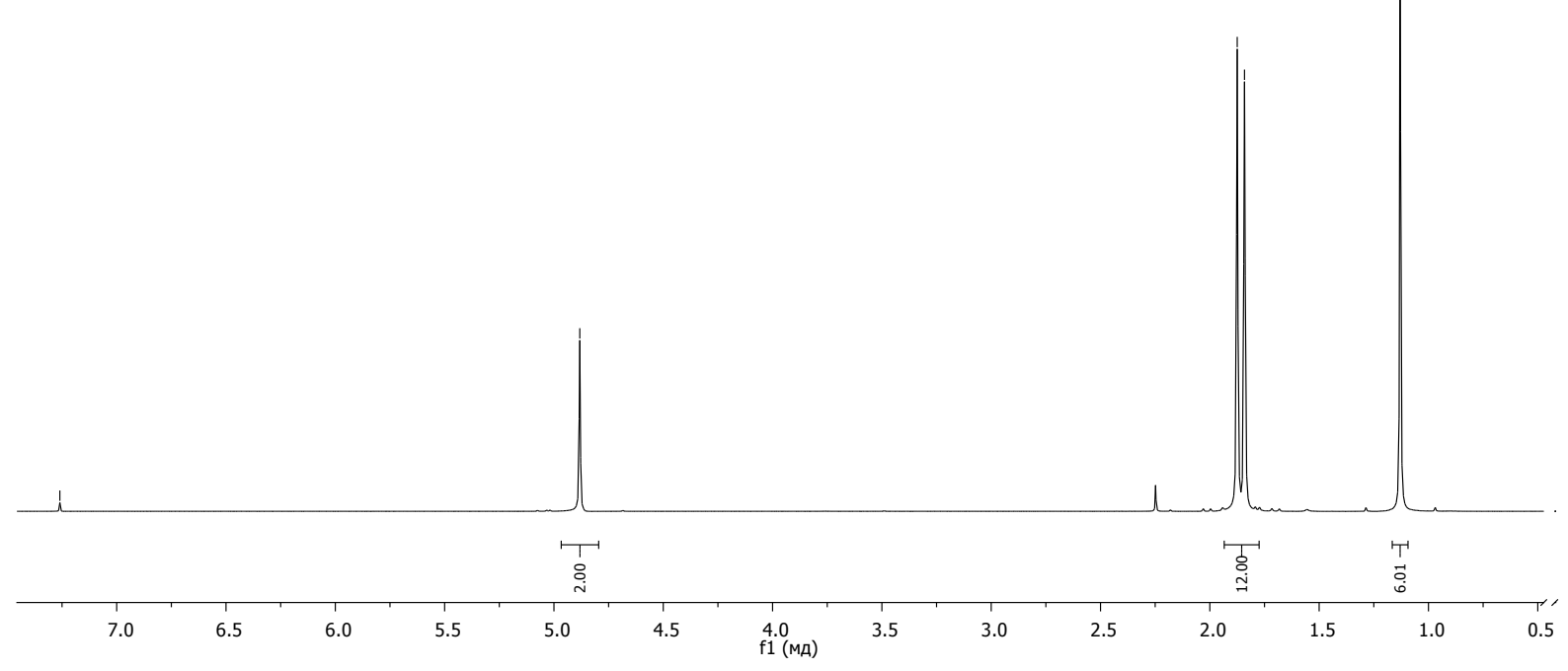

Figure S2. ${ }^{13} \mathrm{C}$ NMR spectrum of 4-methylene-1,1,2,3,5,6-hexamethylcyclohexadiene-2,5 $\mathrm{C}_{6} \mathrm{Me}_{6}\left(\mathrm{CH}_{2}\right)(2)$ in $\mathrm{CDCl}_{3}$<smiles>C=C1C(C)=C(C)C(C)(C)C(C)=C1C</smiles>

2
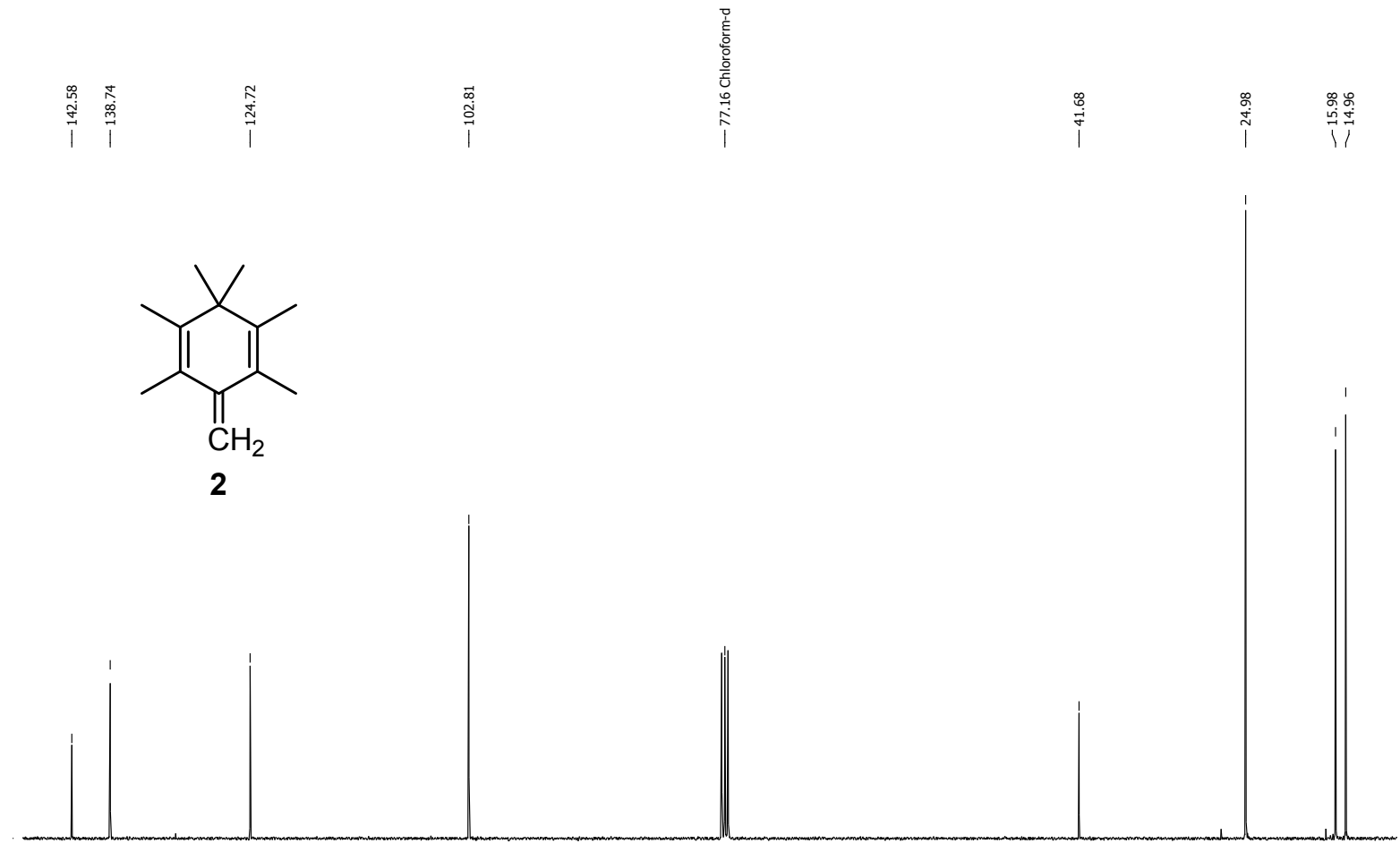

$\begin{array}{llllllllllllllllllllllllllllllllllllll}145 & 140 & 135 & 130 & 125 & 120 & 115 & 110 & 105 & 100 & 95 & 90 & 85 & 80 & 75 & 70 & 65 & 60 & 55 & 50 & 45 & 40 & 35 & 30 & 25 & 20 & 15 & 10\end{array}$ 
Figure S3. ${ }^{1} \mathrm{H}$ NMR spectrum of $\left[\left(\eta^{5}-\mathrm{C}_{6} \mathrm{Me}_{7}\right) \mathrm{RhCl}_{2}\right]_{2}(4)$ in $\mathrm{CDCl}_{3}$ (signal at $3.73 \mathrm{ppm}$ due to residual $1,2-$ dichloroethane)

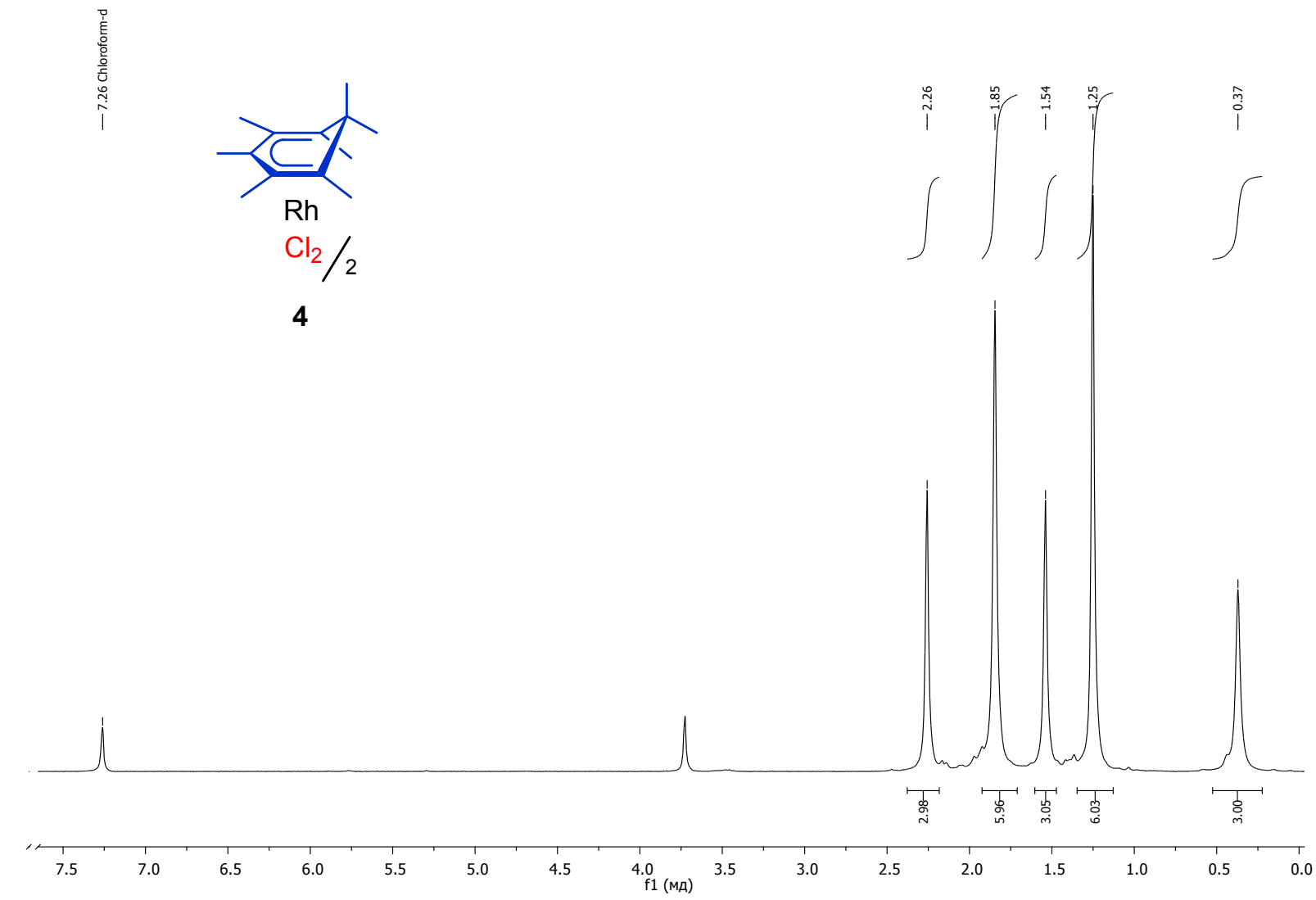


Figure S4. ${ }^{1} \mathrm{H}$ NMR spectrum of $\left[\left(\eta^{6}-\mathrm{C}_{6} \mathrm{Me}_{6}=\mathrm{CH}_{2}\right) \mathrm{Rh}(\operatorname{cod})\right] \mathrm{PF}_{6}(5)$ in acetone-d6. Signals of the cyclooctadiene are broad because of the hindered rotation.

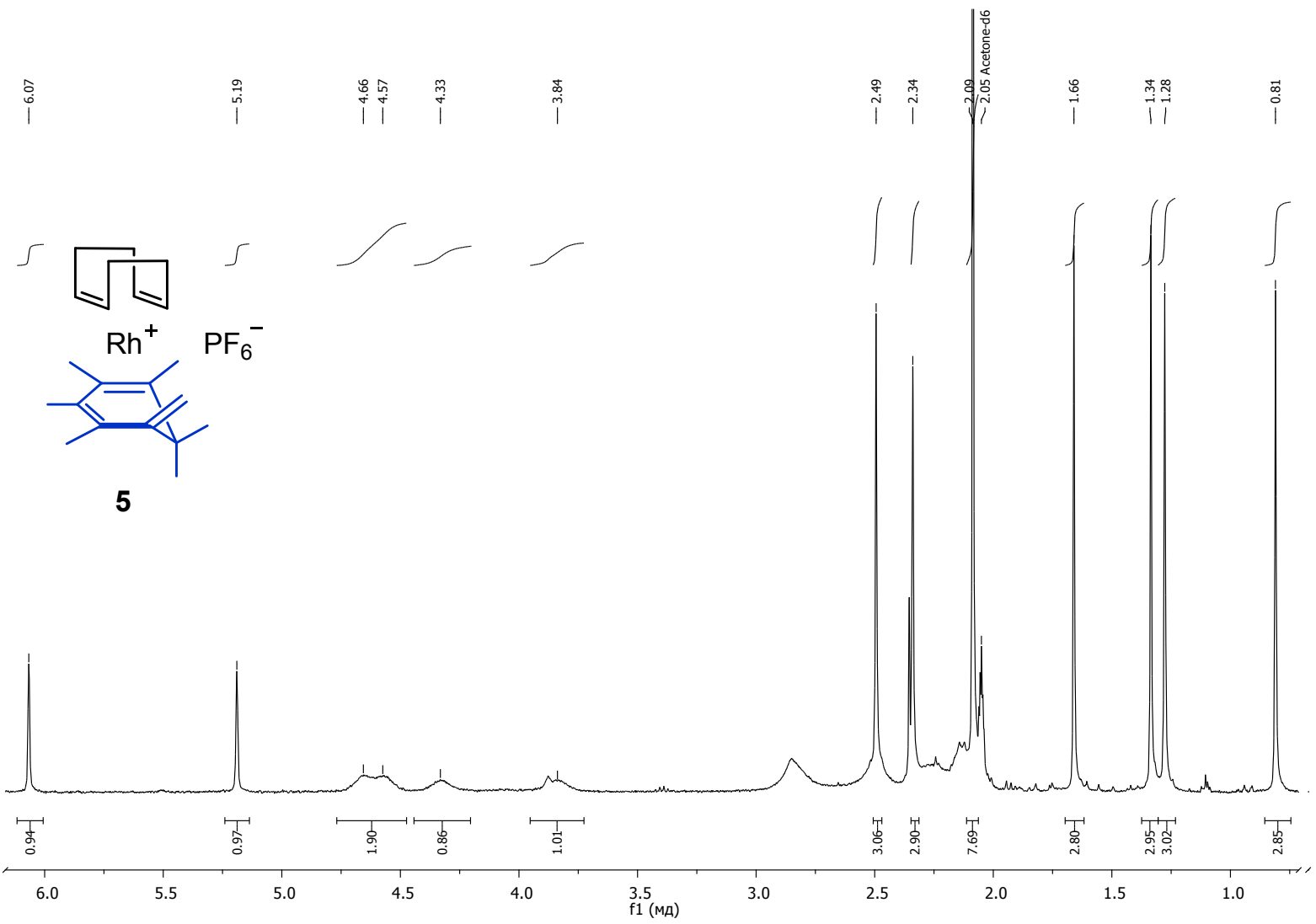

Figure S5. ${ }^{13} \mathrm{C}$ NMR spectrum of $\left[\left(\eta^{6}-\mathrm{C}_{6} \mathrm{Me}_{6}=\mathrm{CH}_{2}\right) \mathrm{Rh}(\operatorname{cod})\right] \mathrm{PF}_{6}(5)$ in acetone-d6. Signals of the cyclooctadiene are broad because of the hindered rotation.

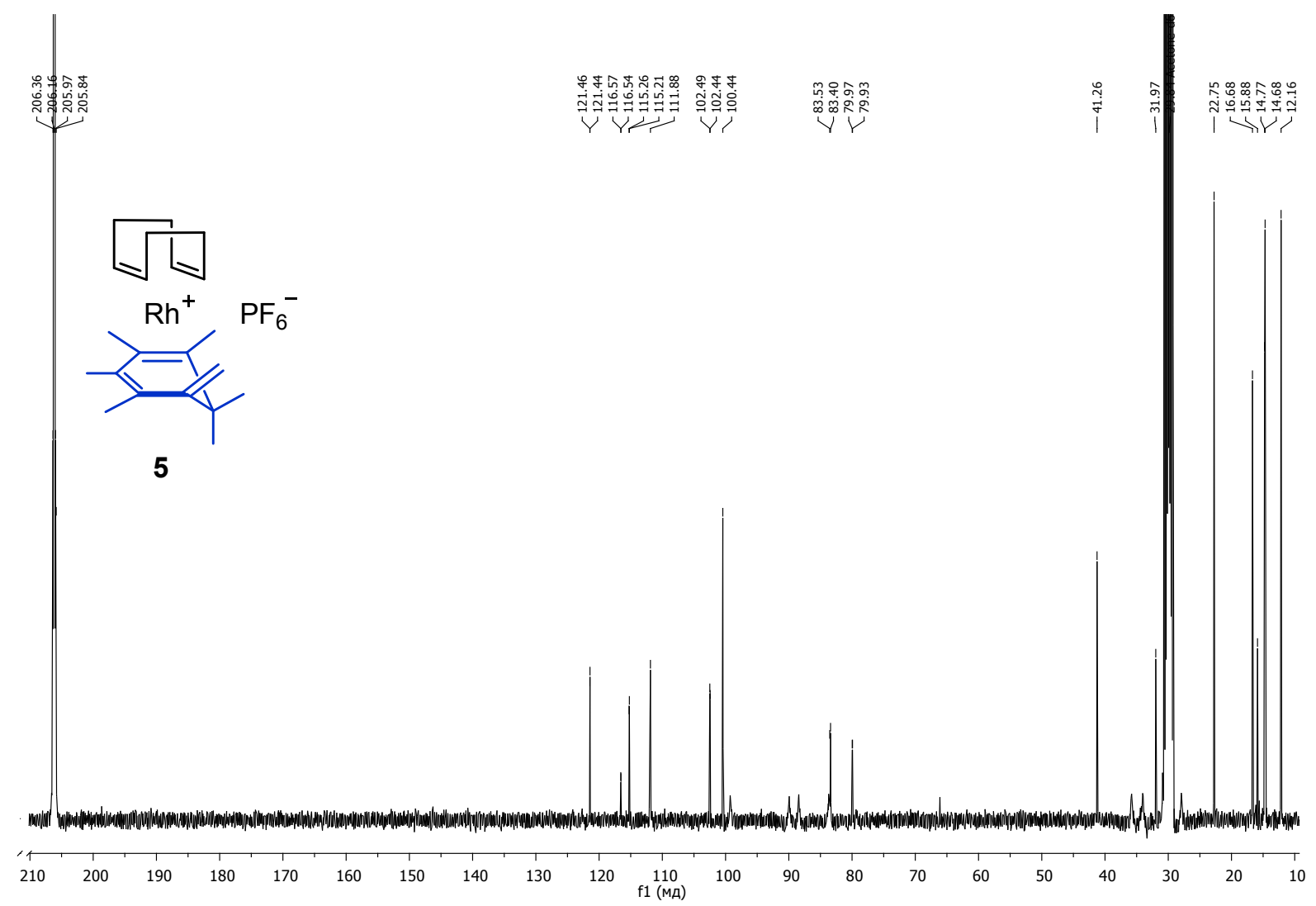


Figure S6. ${ }^{1} \mathrm{H} N M R$ spectrum of $\left(\eta^{5}-\mathrm{C}_{6} \mathrm{Me}_{7}\right) \mathrm{Rh}(\mathrm{Py}) \mathrm{Cl}_{2}(6 \mathrm{6a})$ in $\mathrm{CDCl}_{3}$. Signals of pyridine and some methyl groups are broad because of the hindered rotation.

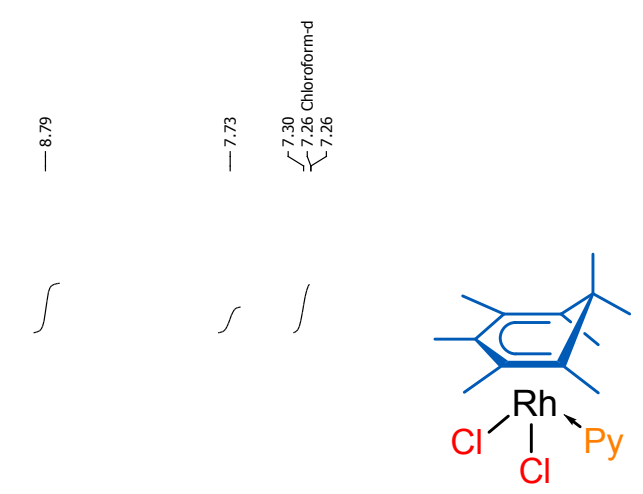

$6 a$

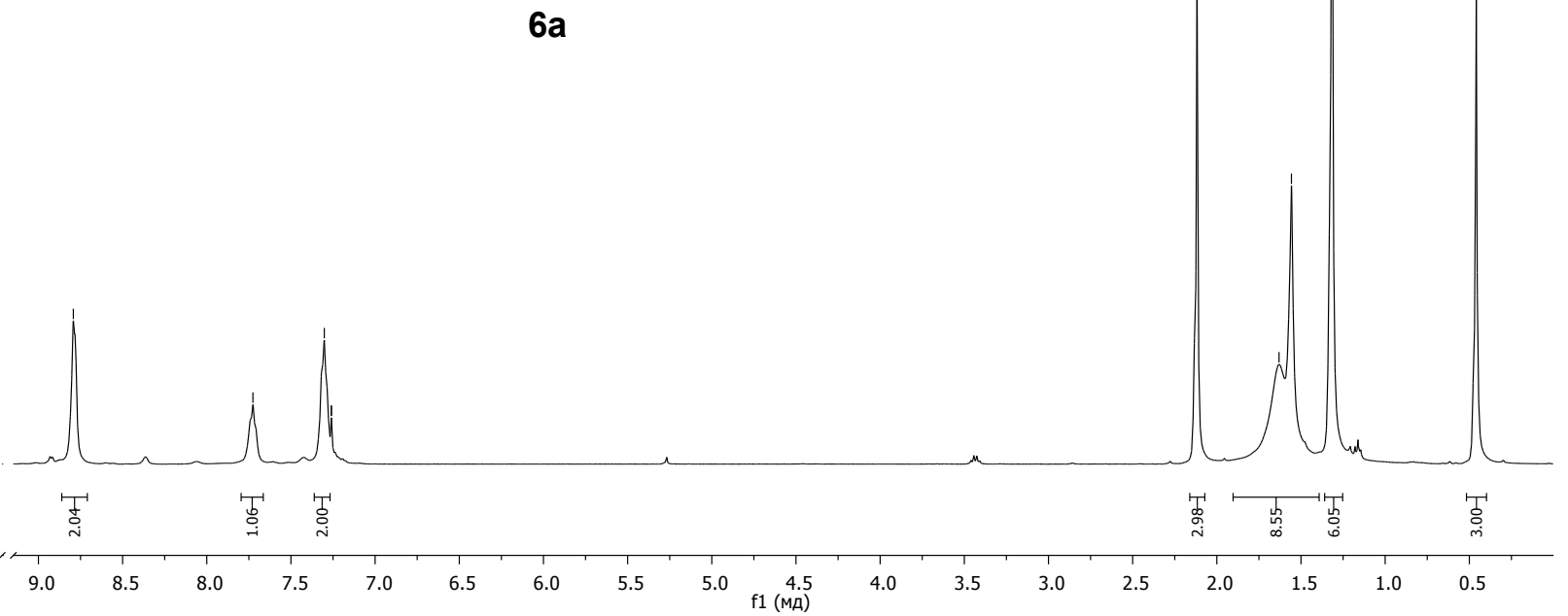

Figure S7. ${ }^{13} \mathrm{C}$ NMR spectrum of $\left(\eta^{5}-\mathrm{C}_{6} \mathrm{Me}_{7}\right) \mathrm{Rh}(\mathrm{Py}) \mathrm{Cl}_{2}(6 \mathbf{a})$ in $\mathrm{CDCl}_{3}$

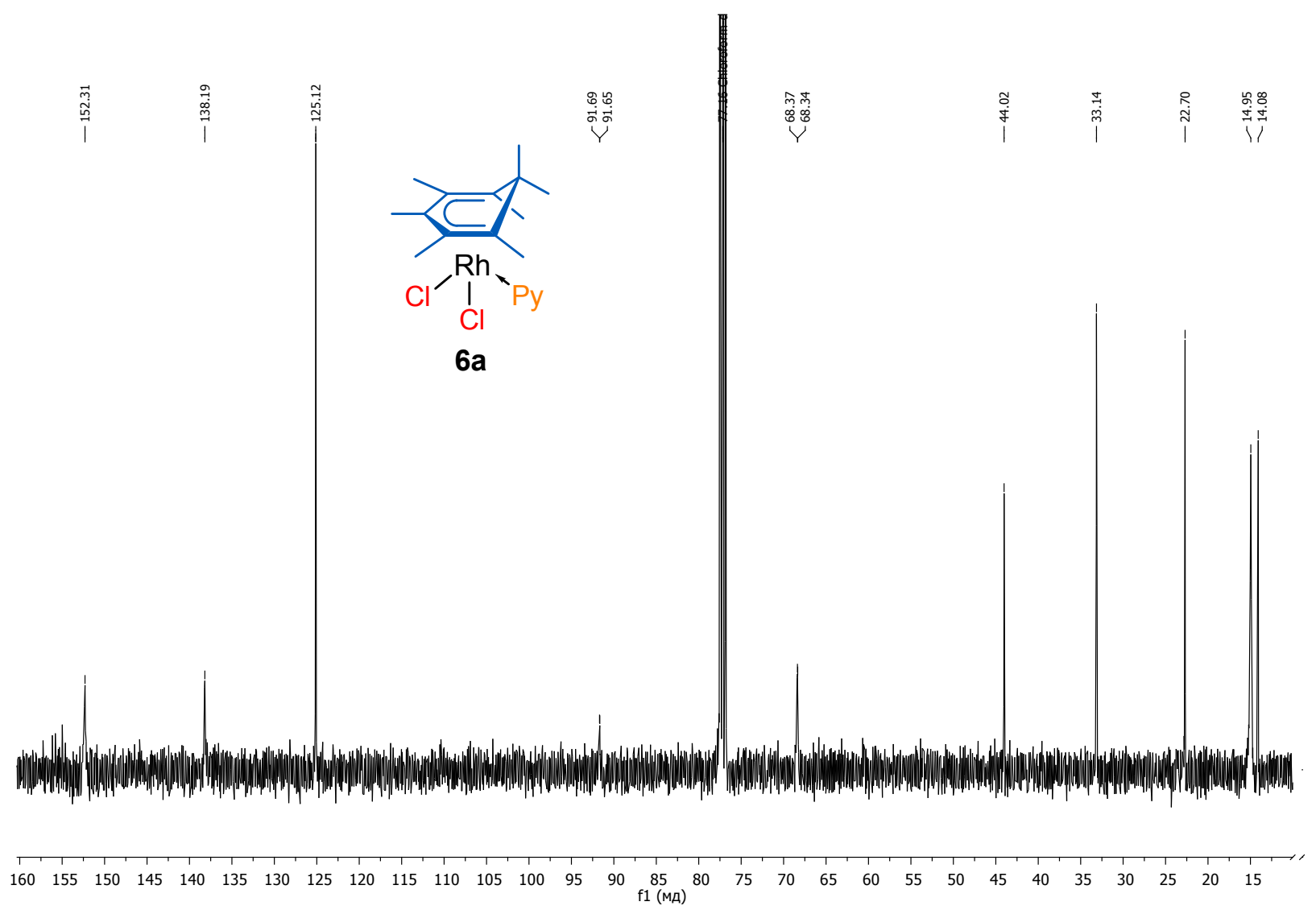


Figure S8. ${ }^{1} \mathrm{H}$ NMR spectrum of $\left(\eta^{5}-\mathrm{C}_{6} \mathrm{Me}_{7}\right) \mathrm{Rh}\left(\mathrm{P}(\mathrm{OEt})_{3}\right) \mathrm{Cl}_{2}(\mathbf{6 b})$ in $\mathrm{CD}_{2} \mathrm{Cl}_{2}$. Signals of some methyl groups are broad because of the hindered rotation.

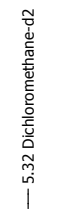

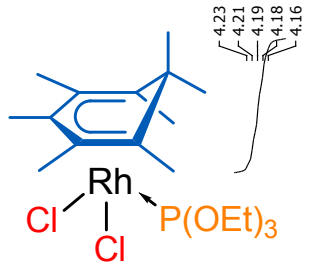

6b
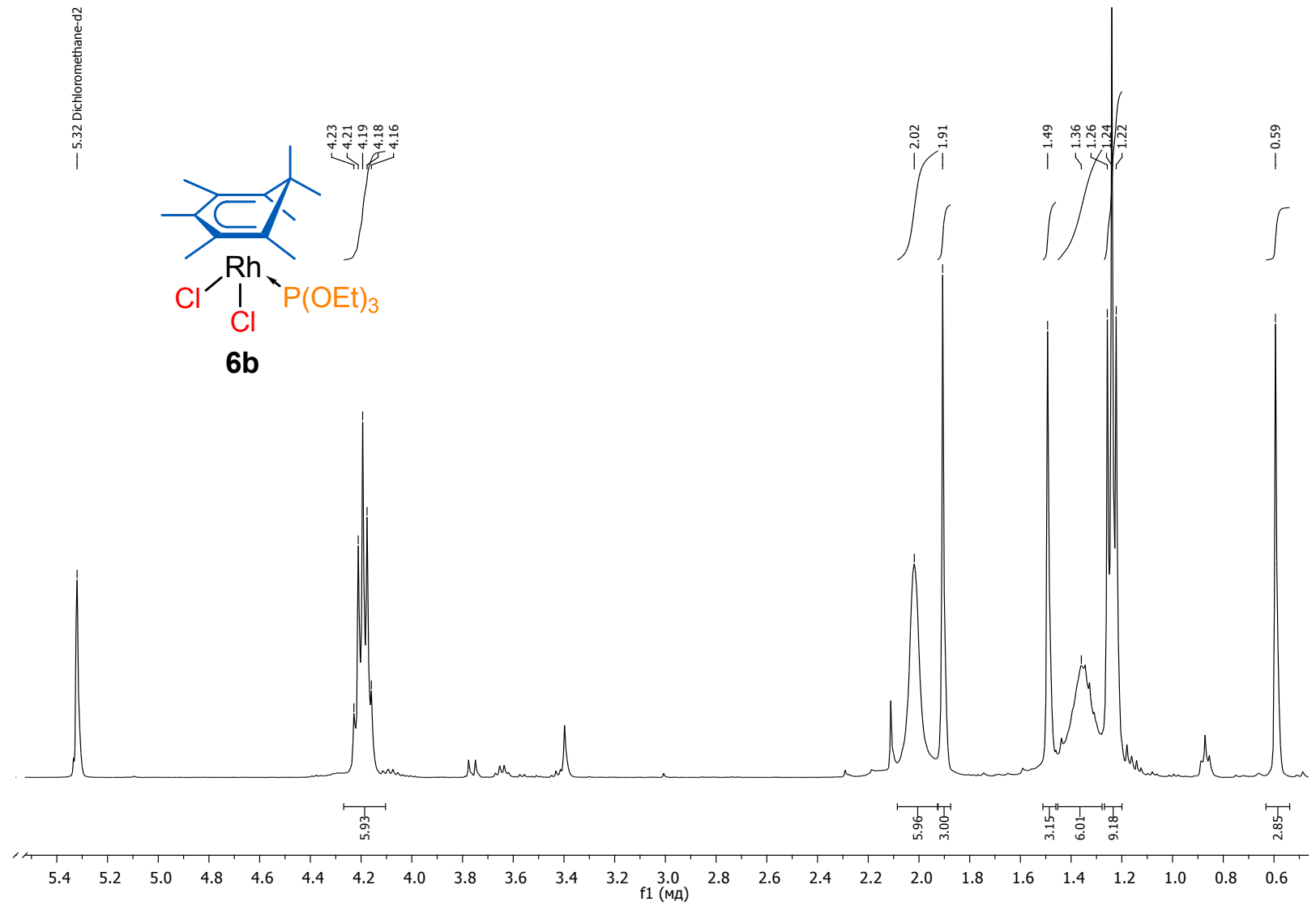

Figure S9. ${ }^{13} \mathrm{C} N M R$ spectrum of $\left(\eta^{5}-\mathrm{C}_{6} \mathrm{Me}_{7}\right) \mathrm{Rh}\left(\mathrm{P}(\mathrm{OEt})_{3}\right) \mathrm{Cl}_{2}(\mathbf{6 b})$ in $\mathrm{CD}_{2} \mathrm{Cl}_{2}$

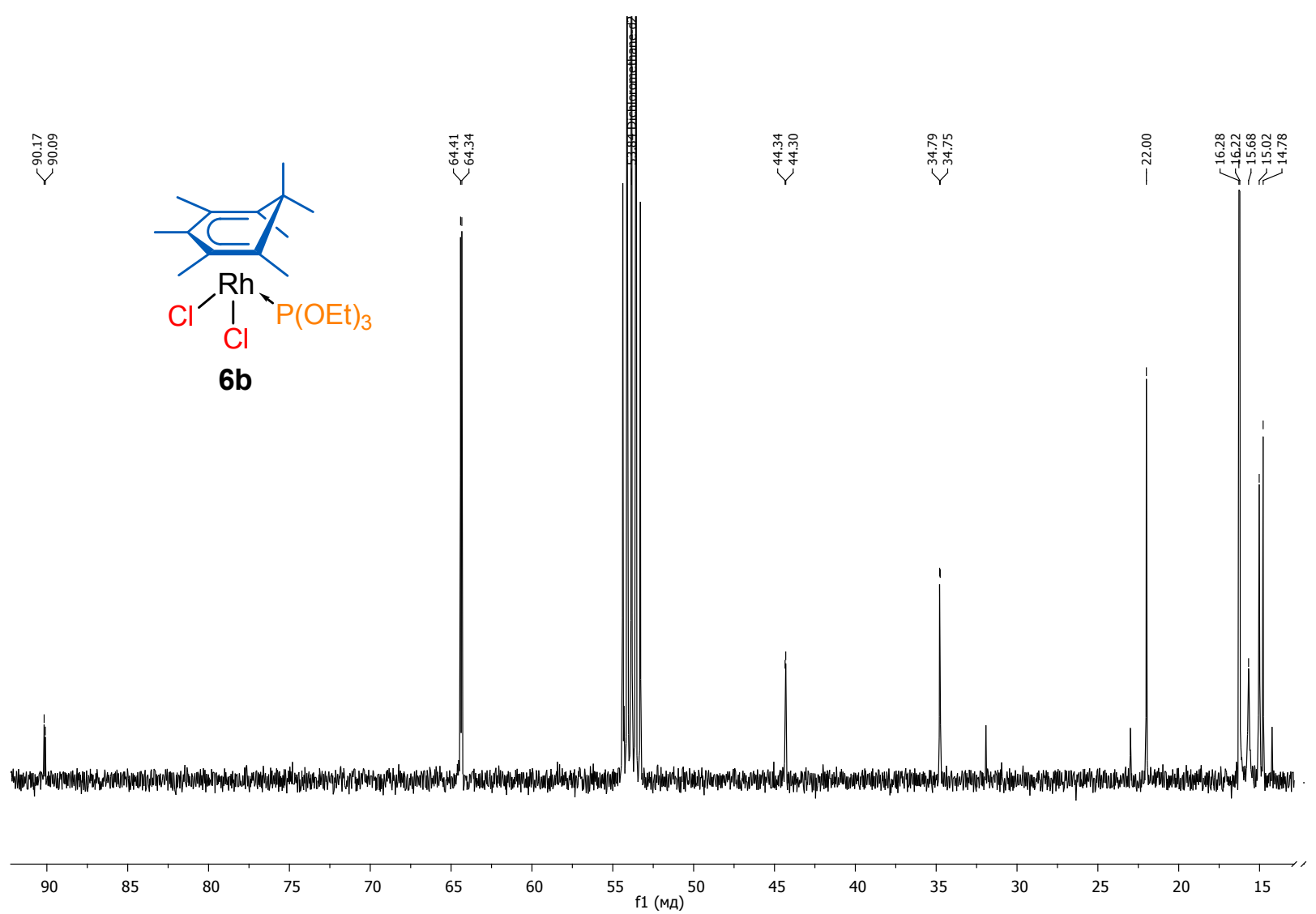


Figure S10. ${ }^{31} \mathrm{P} N M R$ spectrum of $\left(\eta^{5}-\mathrm{C}_{6} \mathrm{Me}_{7}\right) \mathrm{Rh}\left(\mathrm{P}(\mathrm{OEt})_{3}\right) \mathrm{Cl}_{2}(6 \mathbf{b})$ in $\mathrm{CD}_{2} \mathrm{Cl}_{2}$ (traces of $\mathrm{PO}(\mathrm{OEt})_{3}$ present)

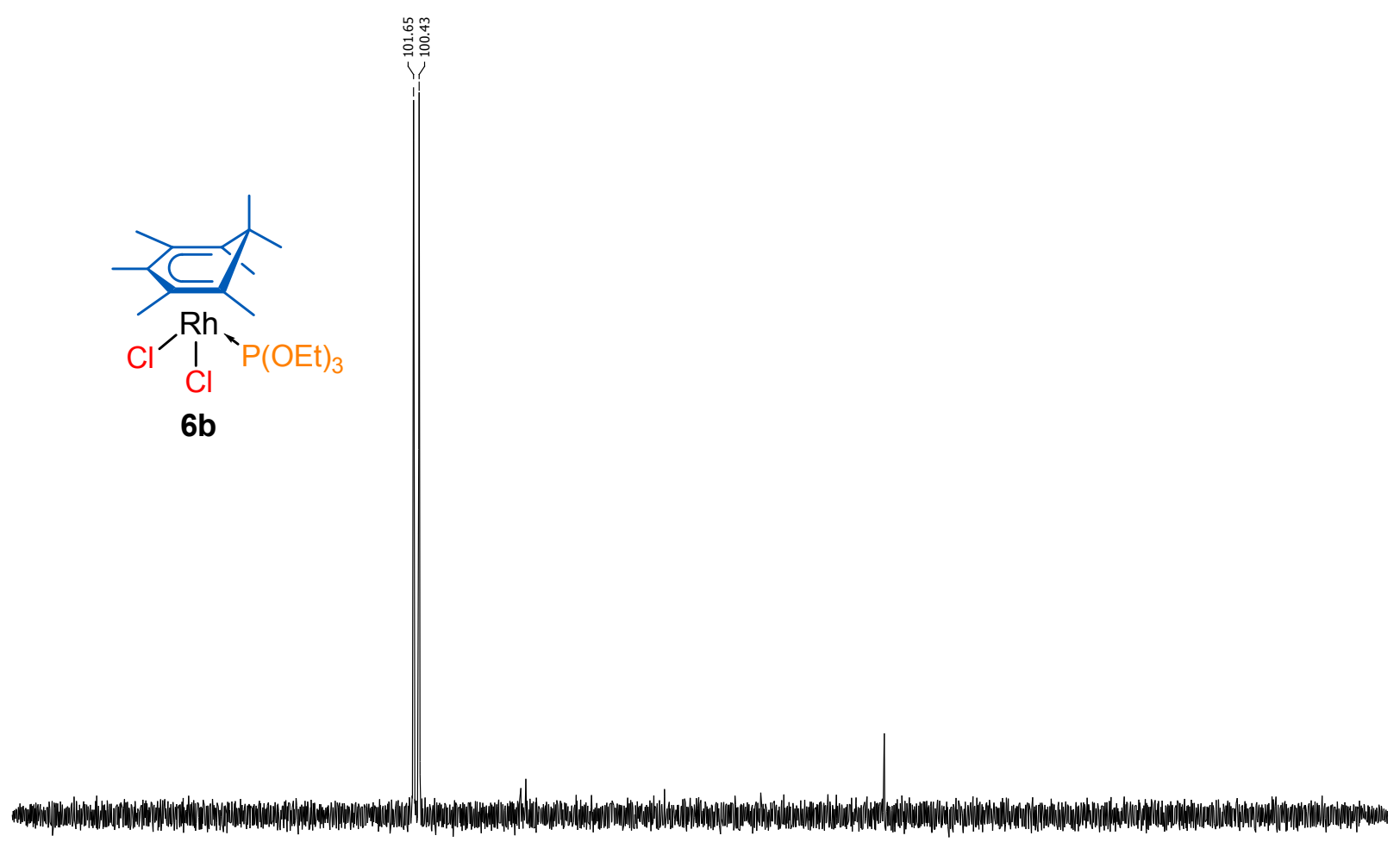

$\begin{array}{lllllllllllllllllllllllllllll}180 & 170 & 160 & 150 & 140 & 130 & 120 & 110 & 100 & 90 & 80 & 70 & 60 & 50 & \underset{\mathrm{f} 1}{40}(\mathrm{MA}) & 30 & 20 & 10 & 0 & -10 & -20 & -30 & -40 & -50 & -60 & -70 & -80 & -90 & -100\end{array}$ 
Figure S11. ${ }^{1} \mathrm{H}$ NMR spectrum of $\left(\eta^{5}-\mathrm{C}_{6} \mathrm{Me}_{7}\right) \mathrm{Rh}\left(\mathrm{PPh}_{3}\right) \mathrm{Cl}_{2}(6 \mathrm{c})$ in $\mathrm{CDCl}_{3}$ (traces of $\mathrm{CH}_{2} \mathrm{Cl}_{2}$ present). Signals are broad because of the hindered rotation.

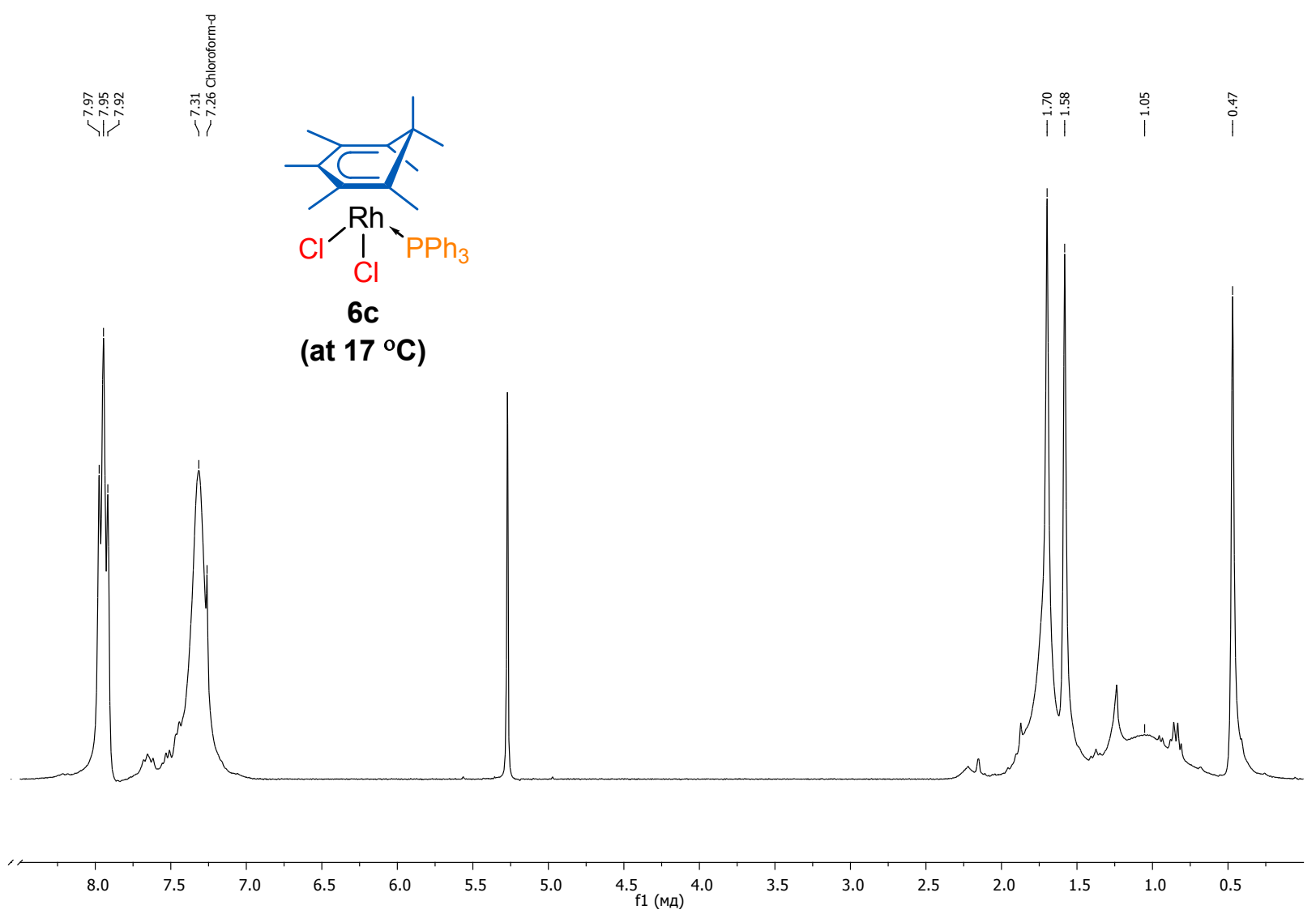

Figure S12. ${ }^{1} \mathrm{H}$ NMR spectrum of $\left(\eta^{5}-\mathrm{C}_{6} \mathrm{Me}_{7}\right) \mathrm{Rh}\left(\mathrm{PPh}_{3}\right) \mathrm{Cl}_{2}(6 \mathrm{c})$ in $\mathrm{CDCl}_{3}$ (traces of $\mathrm{CH}_{2} \mathrm{Cl}_{2}$ present)

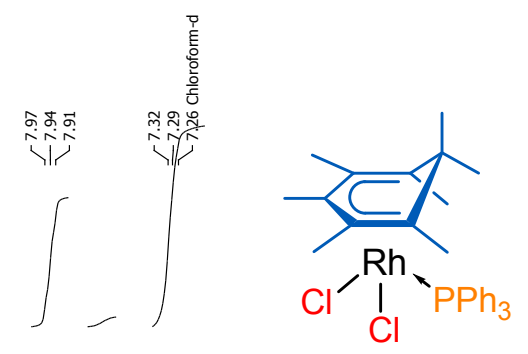

6c

(at $42{ }^{\circ} \mathrm{C}$ )
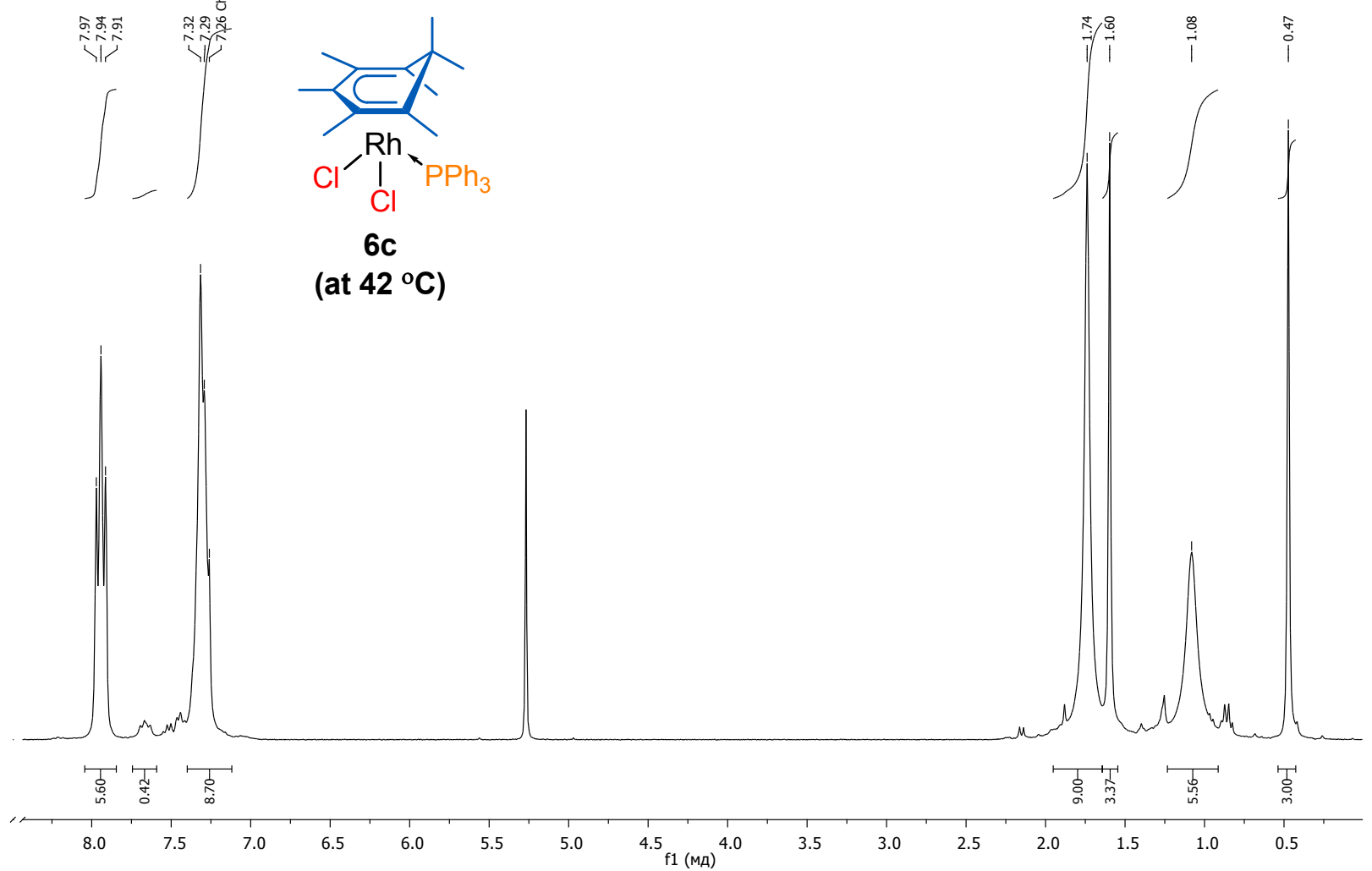
Figure S13. ${ }^{13} \mathrm{C} N M R$ spectrum of $\left(\eta^{5}-\mathrm{C}_{6} \mathrm{Me}_{7}\right) \mathrm{Rh}\left(\mathrm{PPh}_{3}\right) \mathrm{Cl}_{2}(6 \mathrm{c})$ in $\mathrm{CDCl}_{3}$

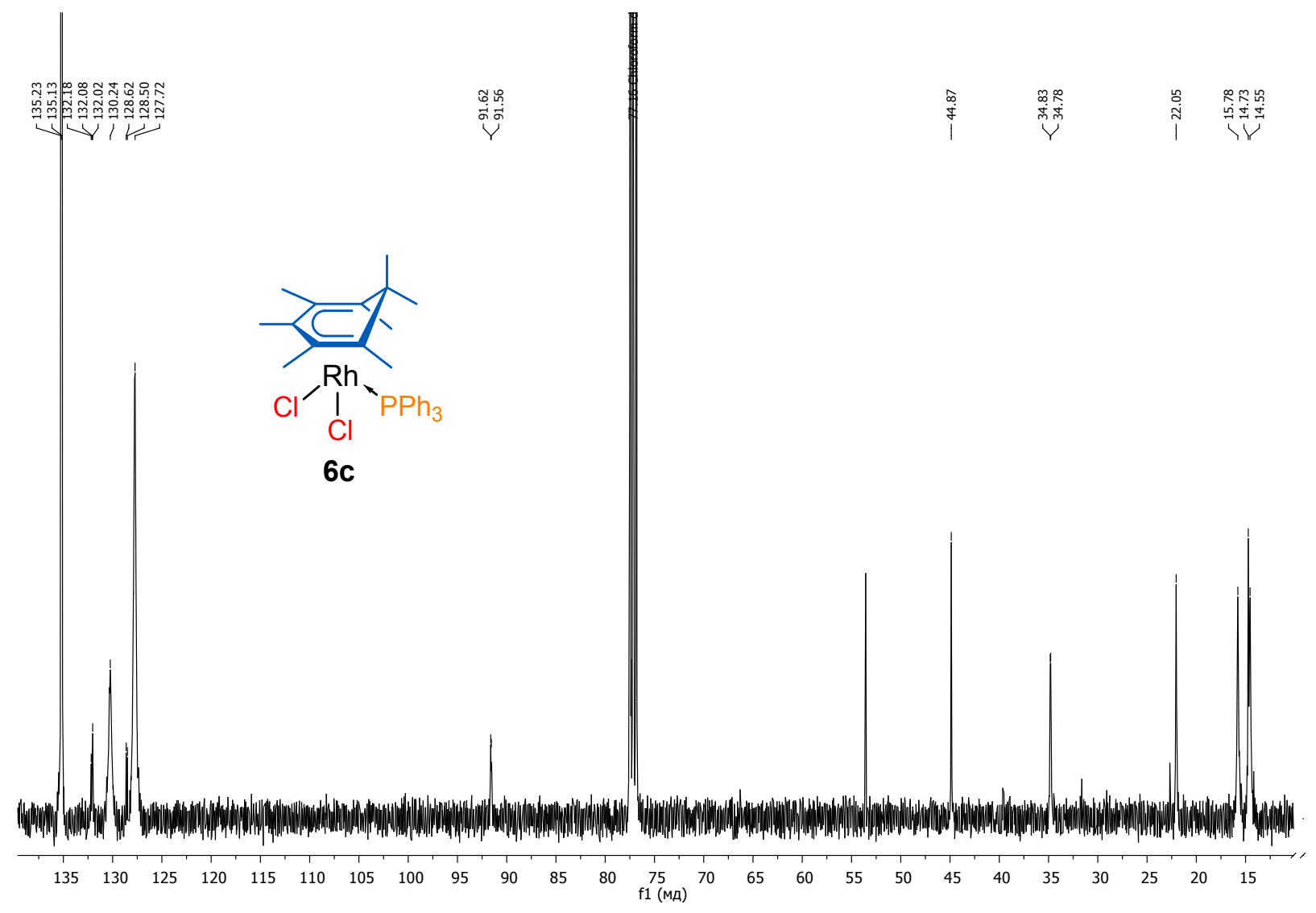

Figure S14. ${ }^{31} \mathrm{P} N M R$ spectrum of $\left[\left(\eta^{5}-\mathrm{C}_{6} \mathrm{Me}_{7}\right) \mathrm{Rh}\left(\mathrm{PPh}_{3}\right) \mathrm{Cl}_{2}\right](6 \mathrm{c})$ in $\mathrm{CDCl}_{3}$

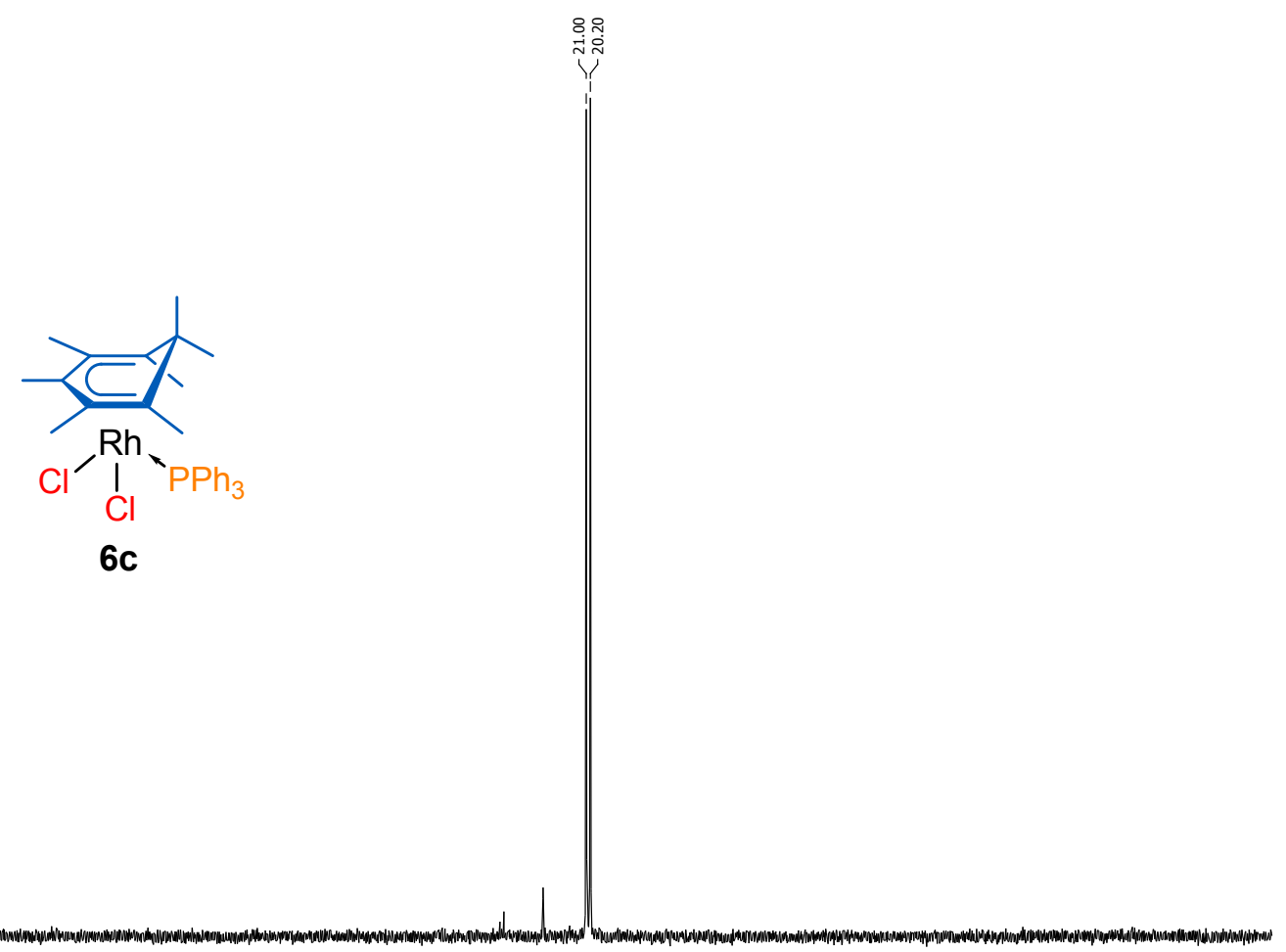

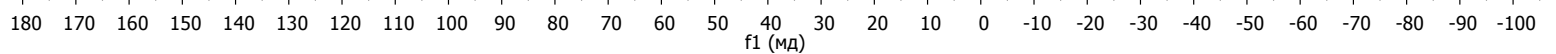


Figure S15. ${ }^{1} \mathrm{H}$ NMR spectrum of $\left[\left(\eta^{5}-\mathrm{C}_{6} \mathrm{Me}_{7}\right) \mathrm{Rh}(\mathrm{dppe}) \mathrm{Cl}\right]\left(\mathrm{PF}_{6}\right)(7)$ in $\mathrm{CDCl}_{3}$ (traces of $\mathrm{Et}_{2} \mathrm{O}$ and $\mathrm{CH}_{2} \mathrm{Cl}_{2}$ present)

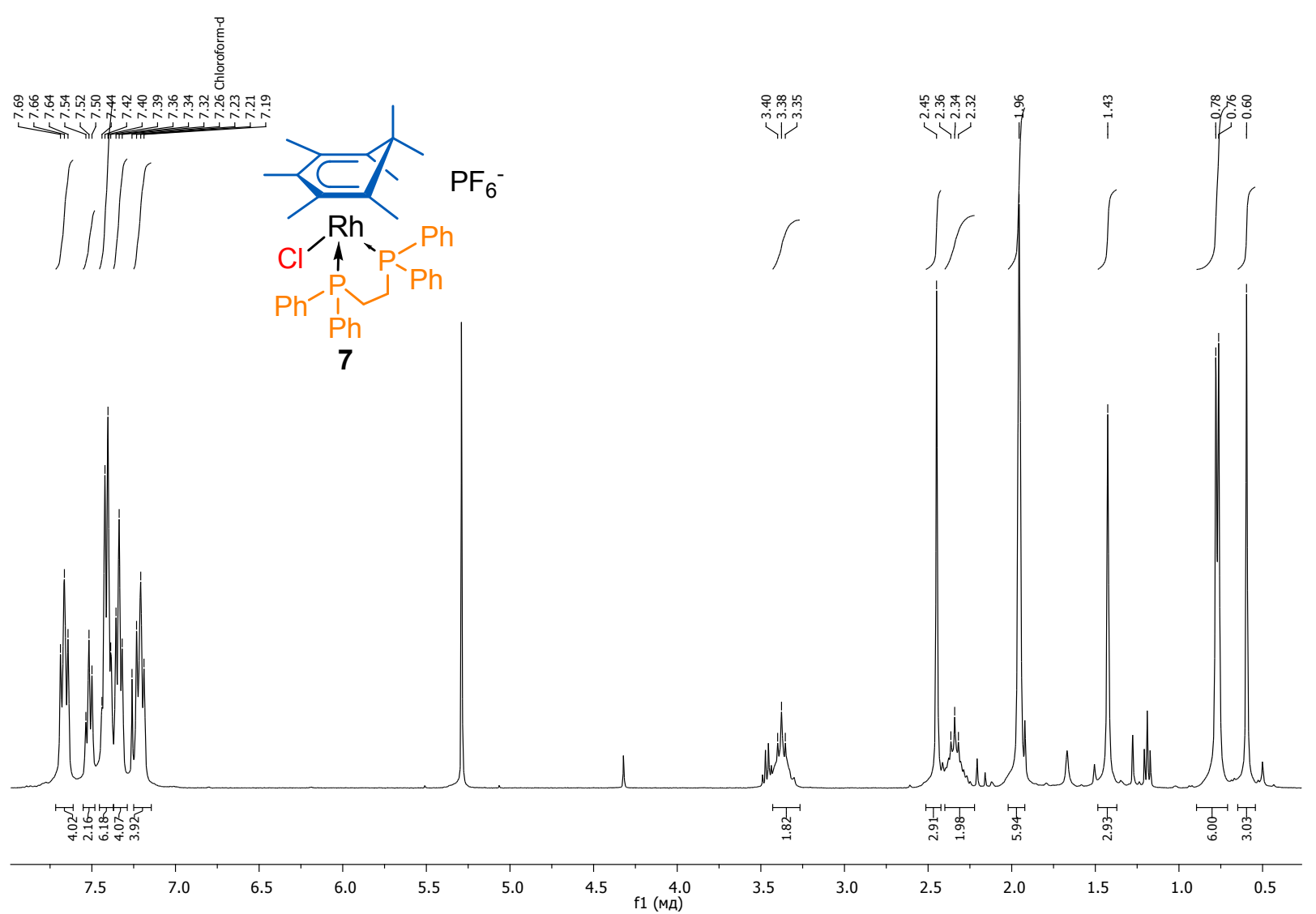

Figure S16. ${ }^{13} \mathrm{C}$ NMR spectrum of $\left[\left(\eta^{5}-\mathrm{C}_{6} \mathrm{Me}_{7}\right) \mathrm{Rh}(\mathrm{dppe}) \mathrm{Cl}\right]\left(\mathrm{PF}_{6}\right)(7)$ in $\mathrm{CDCl}_{3}$

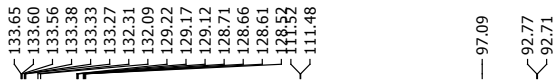
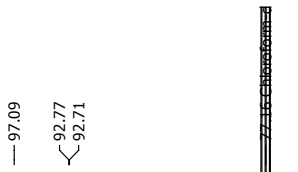

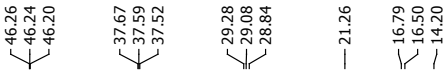
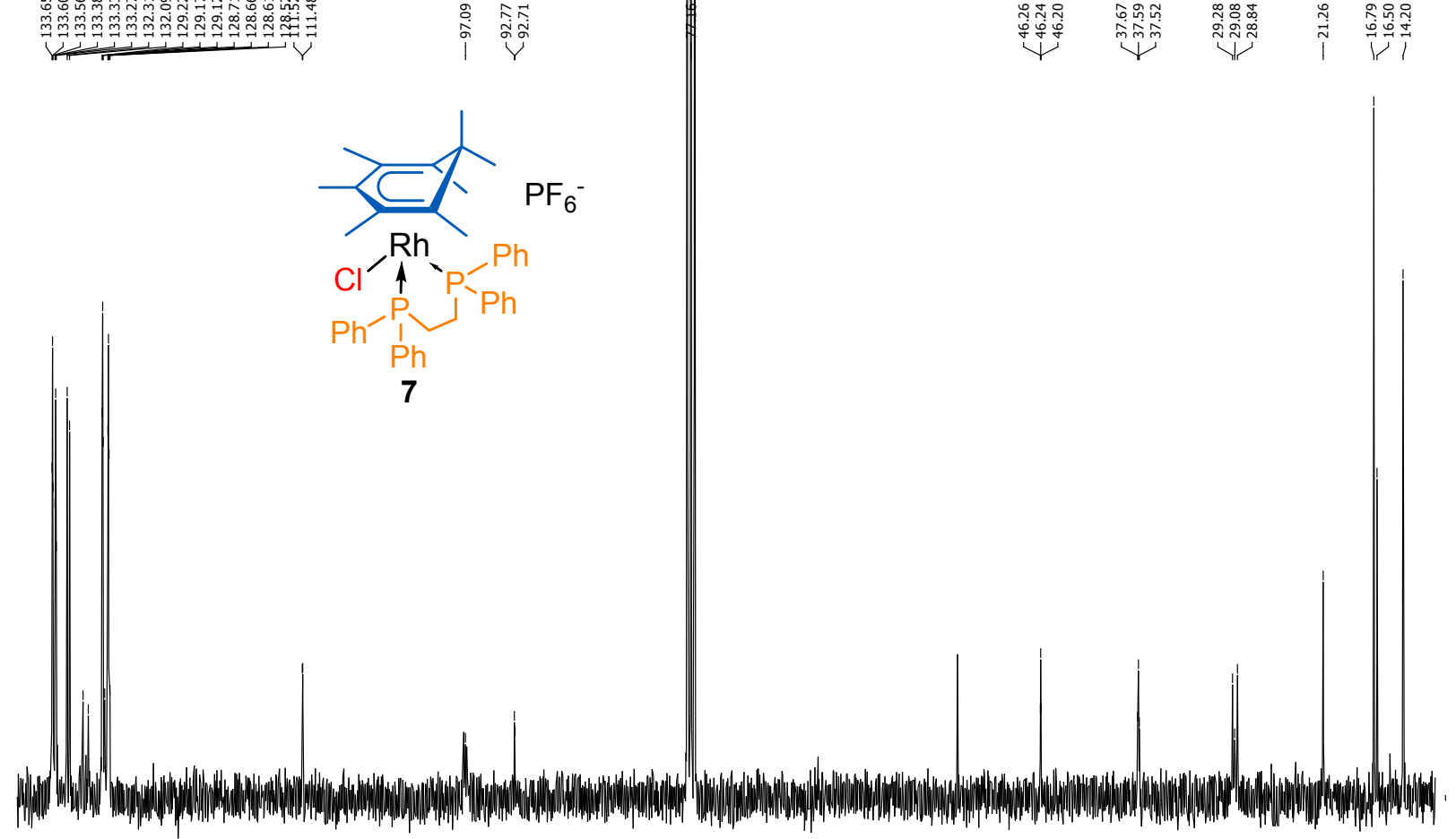

$\begin{array}{lllllllllllllllllllllllllll}135 & 130 & 125 & 120 & 115 & 110 & 105 & 100 & 95 & 90 & 85 & 80 & 75 & 70 & 65 & 60 & 55 & 50 & 45 & 40 & 35 & 30 & 25 & 20 & 15\end{array}$ 
Figure S17. ${ }^{31} \mathrm{P} N M R$ spectrum of $\left[\left(\eta^{5}-\mathrm{C}_{6} \mathrm{Me}_{7}\right) \mathrm{Rh}(\mathrm{dppe}) \mathrm{Cl}\right]\left(\mathrm{PF}_{6}\right)(\mathbf{7})$ in $\mathrm{CDCl}_{3}$

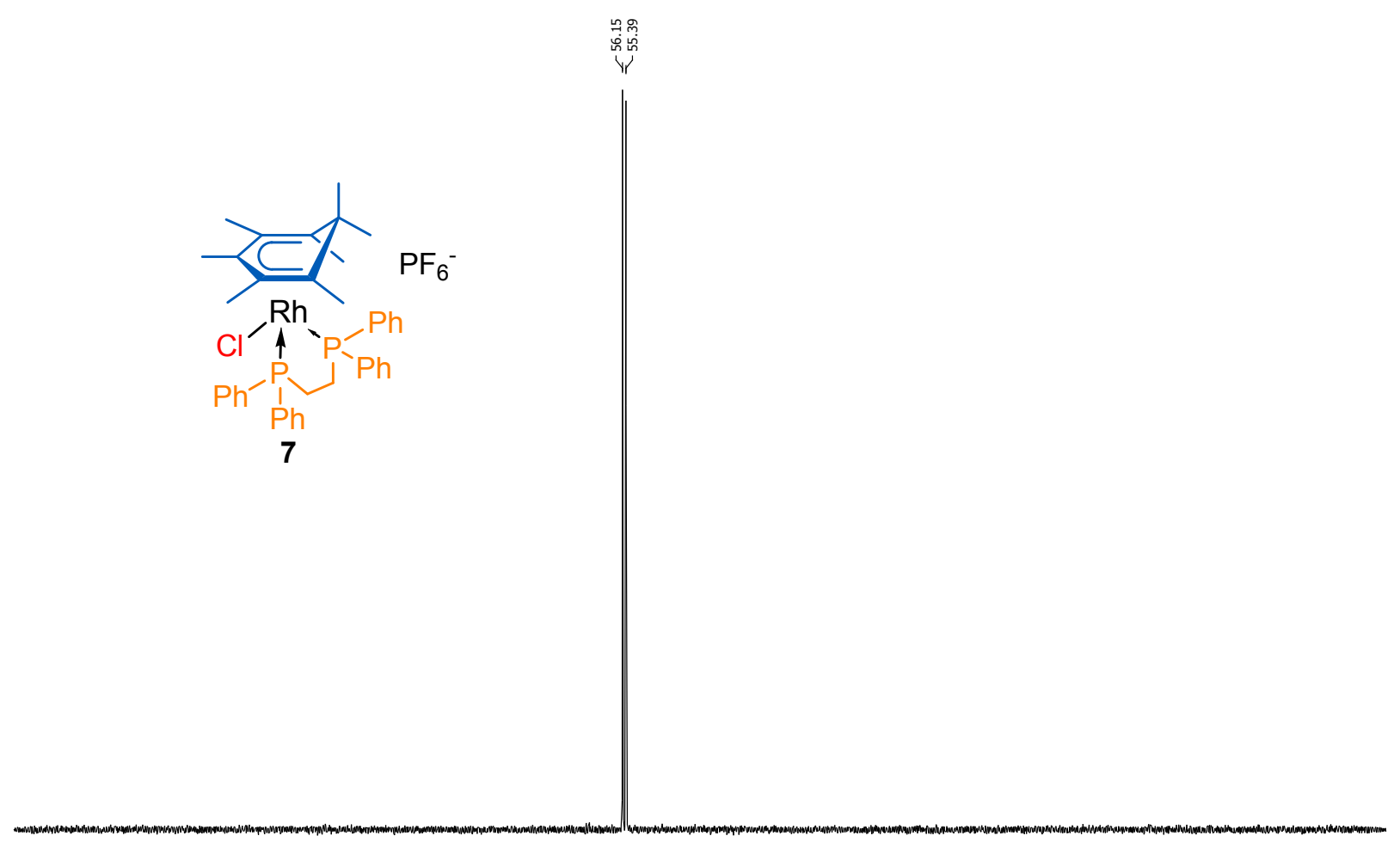

$\begin{array}{lllllllllllllllllllllllllllll}180 & 170 & 160 & 150 & 140 & 130 & 120 & 110 & 100 & 90 & 80 & 70 & 60 & 50 & \begin{array}{l}40 \\ \mathrm{f} 1(\mathrm{MA})\end{array} & 30 & 20 & 10 & 0 & -10 & -20 & -30 & -40 & -50 & -60 & -70 & -80 & -90 & -100\end{array}$ 
Figure S18. ${ }^{1} \mathrm{H}$ NMR spectrum of $\left[\left(\eta^{5}-\mathrm{C}_{6} \mathrm{Me}_{7}\right) \mathrm{Rh}\left(\mathrm{C}_{6} \mathrm{Me}_{6}\right)\right]\left(\mathrm{BF}_{4}\right)_{2}(8)$ in nitrometane-d3

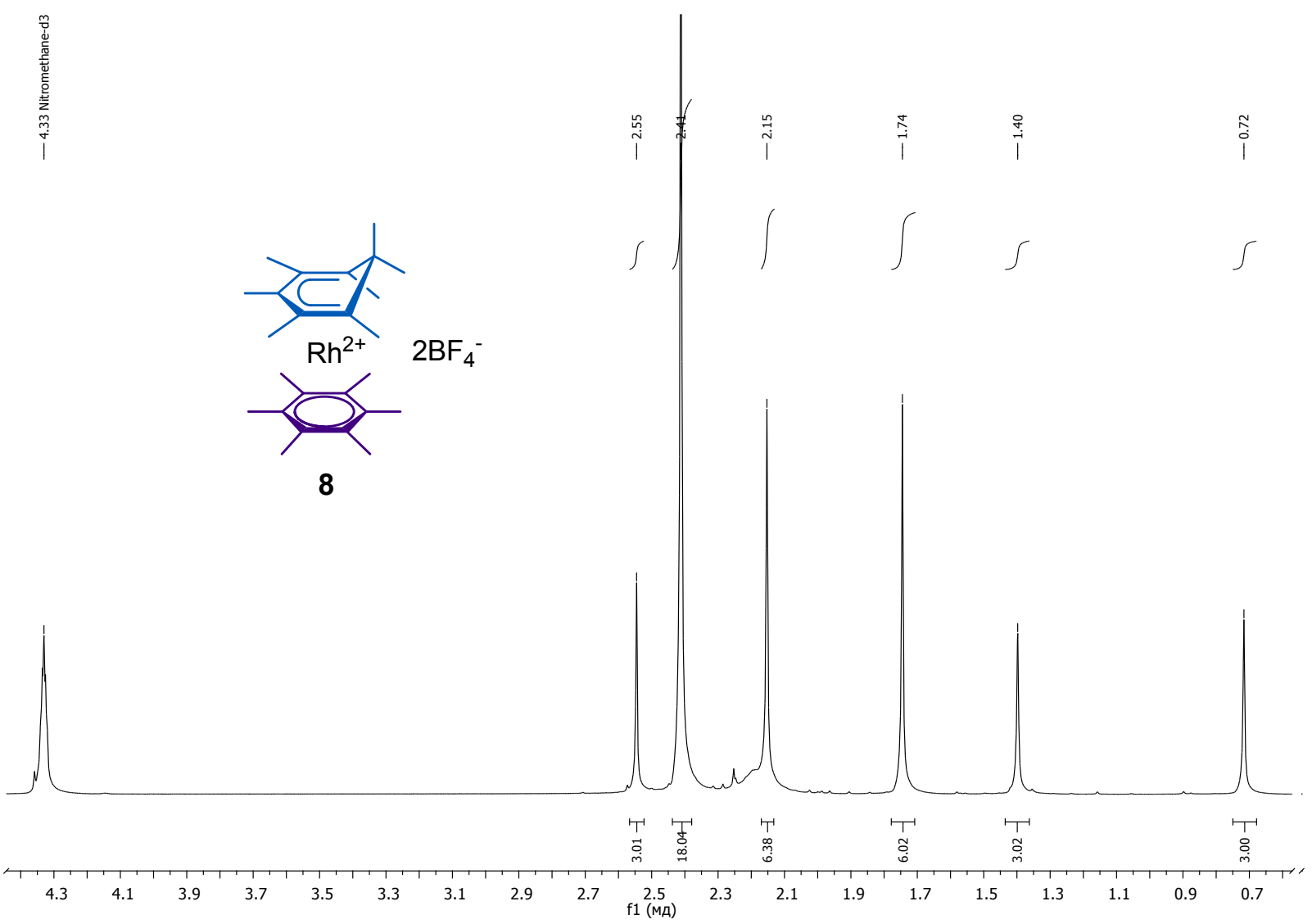

Figure S19. ${ }^{13} \mathrm{C}$ NMR spectrum of $\left[\left(\eta^{5}-\mathrm{C}_{6} \mathrm{Me}_{7}\right) \mathrm{Rh}\left(\mathrm{C}_{6} \mathrm{Me}_{6}\right)\right]\left(\mathrm{BF}_{4}\right)_{2}(8)$ in nitrometane-d3
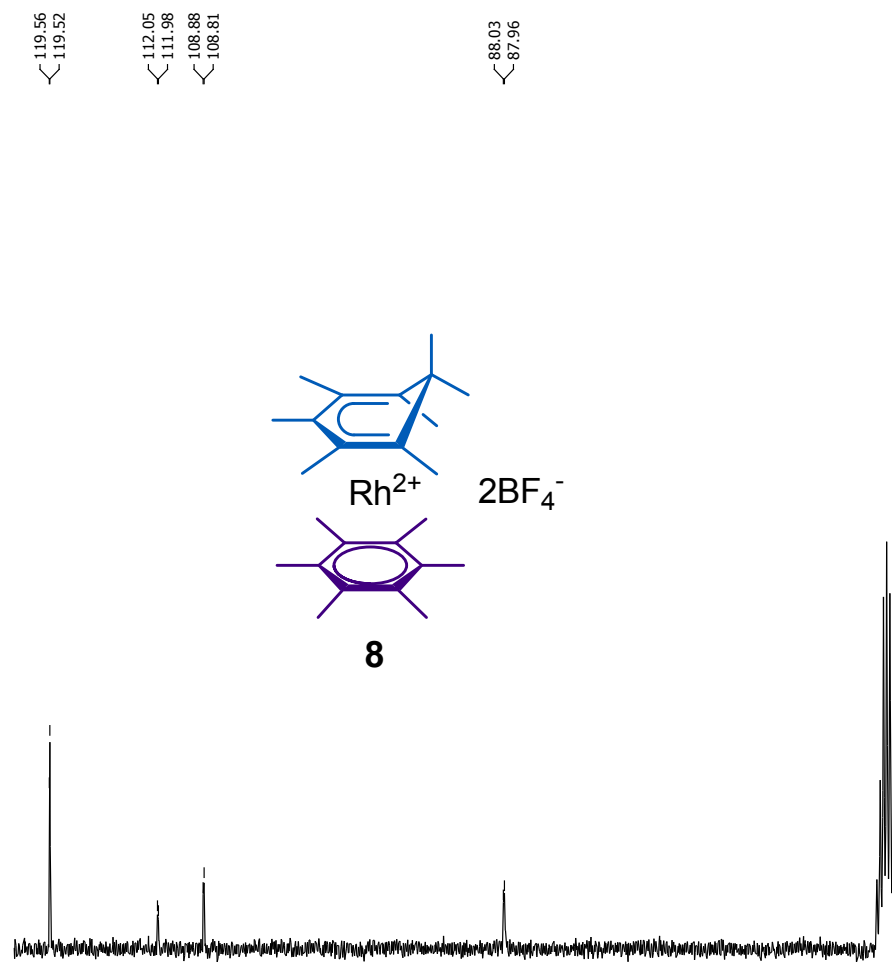
Figure S20. ${ }^{1} \mathrm{H}$ NMR spectrum of $\left(\eta^{5}-\mathrm{C}_{6} \mathrm{Me}_{7}\right) \mathrm{Rh}\left(\mathrm{C}_{6} \mathrm{H}_{4}-\mathrm{Py}\right) \mathrm{Cl}(9)$ in $\mathrm{CDCl}_{3}$

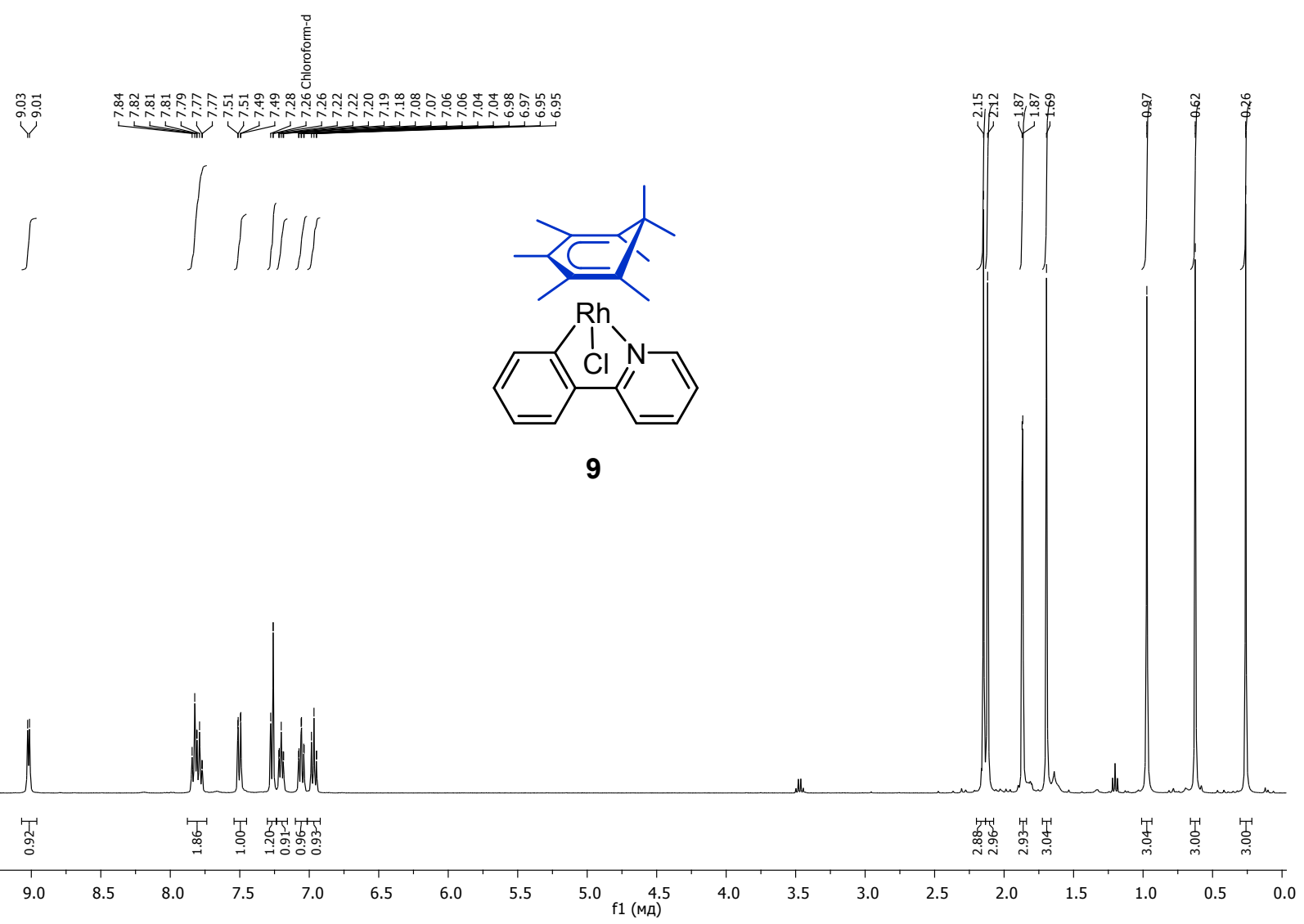

Figure S21. ${ }^{13} \mathrm{C}$ NMR spectrum of $\left(\eta^{5}-\mathrm{C}_{6} \mathrm{Me}_{7}\right) \mathrm{Rh}\left(\mathrm{C}_{6} \mathrm{H}_{4}-\mathrm{Py}\right) \mathrm{Cl}(9)$ in $\mathrm{CDCl}_{3}$

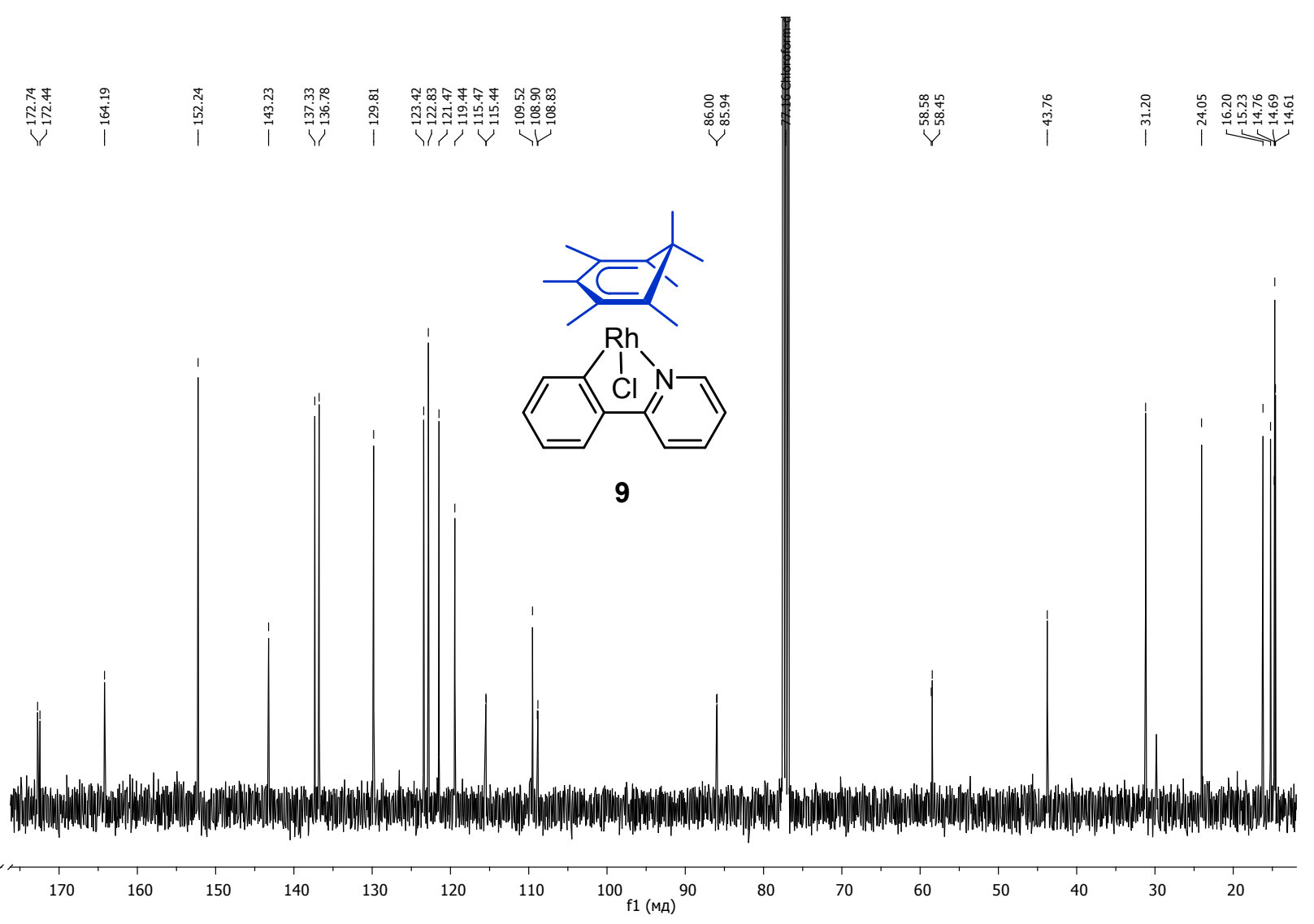


To a mixture of $\left(\eta^{5}-\mathrm{C}_{6} \mathrm{Me}_{7}\right) \mathrm{Rh}\left(\mathrm{C}_{6} \mathrm{H}_{4}-\mathrm{Py}\right) \mathrm{Cl}(9)(10.0 \mathrm{mg}, 0.021 \mathrm{mmol})$ and TIOAc $(6.2 \mathrm{mg}, 0.023 \mathrm{mmol})$ methanol-d6 $(0.6 \mathrm{~mL})$, containing 3-hexyne $(2.6 \mathrm{mg}, 0.021 \mathrm{mmol})$, was added and this suspension was stirred for 1 hour. After sedimentation of $\mathrm{TICl}$, the ${ }^{1} \mathrm{H}$ NMR spectrum was recorded.

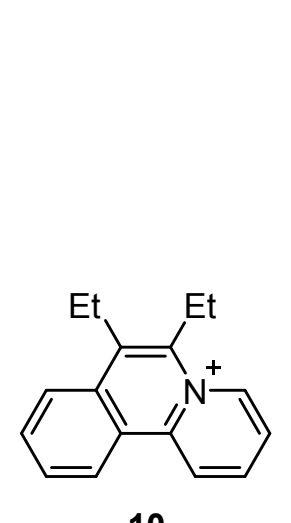

10
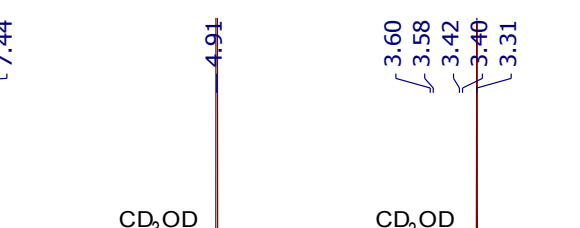

구국유 웅요용 


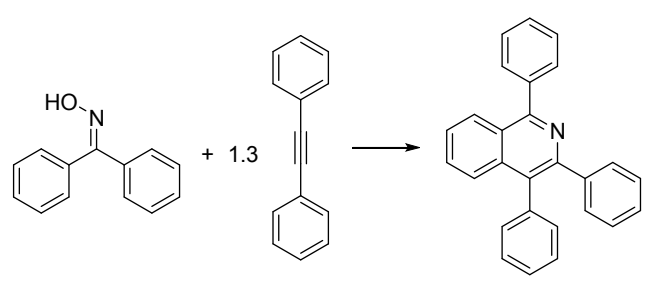

CsOAc (12 mg, 0.061 mmol), [( $\left.\left.\eta^{5}-\mathrm{C}_{6} \mathrm{Me}_{7}\right) \mathrm{RhCl}_{2}\right]_{2}(7 \mathrm{mg}, 0.010 \mathrm{mmol}, 5 \mathrm{~mol} \%)$, benzophenone oxime (40 $\mathrm{mg}, 0.20 \mathrm{mmol}$ ), and diphenylacetylene $(47 \mathrm{mg}, 0.26 \mathrm{mmol}$ ) were placed in a Schlenk tube and methanol $(2 \mathrm{~mL})$ was added. The resulting mixture was stirred at $60^{\circ} \mathrm{C}$ for $16 \mathrm{~h}$. No significant conversion of the starting compounds and formation of the product was detected by TLC. A control experiment with $\left[\mathrm{Cp} * \mathrm{RhCl}_{2}\right]_{2}$ catalyst successfully gave the product in accordance with the literature procedure (Zhang, X.; Chen, D.; Zhao, M.; Zhao, J.; Jia, A.; Li, X. Synthesis of isoquinolines via rhodium(iii)-catalyzed dehydrative $\mathrm{C}-\mathrm{C}$ and $\mathrm{C}-\mathrm{N}$ coupling between oximines and alkynes. Adv. Synth. Catal. 2011, 353, 719723).<smiles>CCCc1ccc(=O)oc1-c1ccccc1-c1ccccc1</smiles>

Maleic acid $(58 \mathrm{mg}, 0.5 \mathrm{mmol})$, diphenylacetylene $(89 \mathrm{mg}, 0.5 \mathrm{mmol}),\left[\left(\eta^{5}-\mathrm{C}_{6} \mathrm{Me}_{7}\right) \mathrm{RhCl}_{2}\right]_{2}(17 \mathrm{mg}, 0.025$ $\mathrm{mmol}, 5 \mathrm{~mol} \%), \mathrm{Ag}_{2} \mathrm{CO}_{3}(138 \mathrm{mg}, 0.5 \mathrm{mmol}$ ) were placed in a Schlenk tube and DMF (2.5 mL) was added. The resulting mixture was stirred under argon at $120^{\circ} \mathrm{C}$ for $6 \mathrm{~h}$. No significant conversion of the starting compounds and formation of the product was detected by TLC. A control experiment with $\left[\mathrm{Cp}^{*} \mathrm{RhCl}_{2}\right]_{2}$ catalyst successfully gave the product in accordance with the literature procedure (Itoh, M.; Shimizu, M.; Hirano, K.; Satoh, T.; Miura, M. rhodium-catalyzed decarboxylative and dehydrogenative coupling of maleic acids with alkynes and alkenes. J. Org. Chem. 2013, 78, 11427-11432).<smiles>CC(=O)n1c(-c2ccccc2)c(-c2ccccc2)c2ccccc21</smiles>

$\mathrm{AgPF}_{6}(15 \mathrm{mg}, 0.059 \mathrm{mmol}),\left[\left(\eta^{5}-\mathrm{C}_{6} \mathrm{Me}_{7}\right) \mathrm{RhCl}_{2}\right]_{2}(10 \mathrm{mg}, 0.015 \mathrm{mmol}, 5 \mathrm{~mol} \%), \mathrm{Cu}(\mathrm{OAc})_{2} \cdot \mathrm{H}_{2} \mathrm{O}(125 \mathrm{mg}$, $0.622 \mathrm{mmol}$ ), acetanilide ( $40 \mathrm{mg}, 0.296 \mathrm{mmol})$, and diphenylacetylene $(58 \mathrm{mg}, 0.326 \mathrm{mmol}$ ) were placed in a Schlenk tube and t-BuOH $(2 \mathrm{~mL})$ was added. The the mixture stirred at $80^{\circ} \mathrm{C}$ under air atmosphere for $72 \mathrm{~h}$. No significant conversion of the starting compounds and formation of the product was detected by TLC. A control experiment with $\left[\mathrm{Cp}^{*} \mathrm{RhCl}_{2}\right]_{2}$ catalyst successfully gave the product in accordance with the literature procedure (Hoshino, Y.; Shibata, Y.; Tanaka, K. Oxidative annulation of anilides with internal alkynes using an (electron-deficient $\eta^{5}$-cyclopentadienyl)rhodium(III) catalyst under ambient conditions. Adv. Synth. Catal. 2014, 356, 1577-1585). 


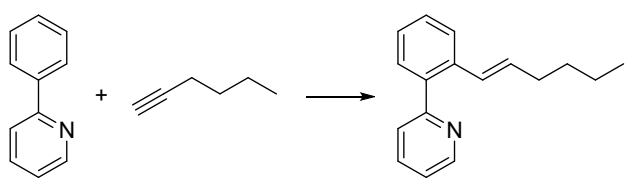

$\mathrm{AgPF}_{6}(15 \mathrm{mg}, 0.059 \mathrm{mmol}),\left[\left(\eta^{5}-\mathrm{C}_{6} \mathrm{Me}_{7}\right) \mathrm{RhCl}_{2}\right]_{2}$ (10 mg, $\left.0.015 \mathrm{mmol}, 5 \mathrm{~mol} \%\right), 2$-phenylpyridine (89 mg, $0.570 \mathrm{mmol})$, and 1-hexyne $(50 \mathrm{mg}, 0.6 \mathrm{mmol})$ were placed in a Schlenk tube and AcOH (1 mL) was added. The mixture was then stirred at $20{ }^{\circ} \mathrm{C}$ for $24 \mathrm{~h}$. No significant conversion of the starting compounds and formation of the product was detected by TLC. A control experiment with $\left[\mathrm{Cp}^{*} \mathrm{RhCl}_{2}\right]_{2}$ catalyst successfully gave the product in accordance with the literature procedure (Duan, C.-L.; Liu, X.-Y.; Tan, Y.-X.; Ding, R.; Yang, S.; Tian, P.; Lin, G.-Q. Acetic acid-promoted rhodium(III)-catalyzed hydroarylation of terminal alkynes. Synlett 2019, 30, 932-938).

Table S1. Crystal data and structure refinement parameters for 2, 5, 6c and $\mathbf{9 .}$

\begin{tabular}{|c|c|c|c|c|}
\hline & 2 & 5 & $6 c$ & 9 \\
\hline Formula unit & $\mathrm{C}_{13} \mathrm{H}_{20}$ & $\mathrm{C}_{25} \mathrm{H}_{54} \mathrm{~B}_{18} \mathrm{CoRh}$ & $\mathrm{C}_{32} \mathrm{H}_{37} \mathrm{Cl}_{5} \mathrm{PRh}$ & $\mathrm{C}_{24} \mathrm{H}_{29} \mathrm{ClNRh}$ \\
\hline Formula weight & 176.30 & 711.38 & 732.79 & 469.86 \\
\hline Crystal system & Monoclinic & Monoclinic & Monoclinic & Monoclinic \\
\hline Space group & $\mathrm{P} 2_{1} / \mathrm{m}$ & $\mathrm{P} 2{ }_{1} / \mathrm{c}$ & $\mathrm{P} 2_{1} / \mathrm{c}$ & $\mathrm{P} 2{ }_{1} / \mathrm{c}$ \\
\hline Z & 2 & 4 & 4 & 4 \\
\hline $\mathrm{a}, \AA$ & $8.661(5)$ & $17.7858(9)$ & $21.3602(10)$ & $11.723(2)$ \\
\hline$b, \AA$ & $7.203(4)$ & $13.3317(7)$ & $16.4884(8)$ & $8.8746(18)$ \\
\hline$c, \AA$ & $8.887(5)$ & $19.7925(12)$ & $22.0602(11)$ & $19.522(4)$ \\
\hline$\alpha,{ }^{\circ}$ & 90 & 90 & 90 & 90 \\
\hline$\beta$, & $91.878(11)$ & $133.4938(10)$ & $155.6948(10)$ & $96.726(5)$ \\
\hline$\gamma, \stackrel{\circ}{ }$ & 90 & 90 & 90 & 90 \\
\hline$V, \AA^{3}$ & $554.1(6)$ & $3404.6(3)$ & $3197.9(3)$ & $2017.0(7)$ \\
\hline$D_{\text {calc }}\left(\mathrm{g} \mathrm{cm}^{-1}\right)$ & 1.057 & 1.387 & 1.522 & 1.547 \\
\hline Linear absorption, $\mu\left(\mathrm{cm}^{-1}\right)$ & 0.58 & 9.92 & 10.23 & 9.88 \\
\hline$F(000)$ & 196 & 1461 & 1495 & 964 \\
\hline $2 \Theta_{\max ,^{\circ}}^{\circ}$ & 52 & 52 & 58 & 54 \\
\hline Reflections measured & 12293 & 46064 & 19276 & 27082 \\
\hline Independent reflections & 1162 & 6709 & 8647 & 4878 \\
\hline Observed reflections $[I>2 \sigma(I)]$ & 867 & 5607 & 8353 & 3330 \\
\hline Parameters & 82 & 412 & 360 & 251 \\
\hline R1 & 0.0986 & 0.0441 & 0.0328 & 0.0419 \\
\hline wR2 & 0.3395 & 0.1179 & 0.1094 & 0.0873 \\
\hline GOF & 1.084 & 1.060 & 1.015 & 0.982 \\
\hline$\Delta \rho_{\max } / \Delta \rho_{\min }\left(\mathrm{e} \AA^{-3}\right)$ & $0.735 /-0.340$ & $3.141 /-1.345$ & $0.565 /-0.547$ & $1.3238 /-1.2281$ \\
\hline
\end{tabular}

